\title{
A historical perspective on ankle ligaments reconstructive surgery
}

\author{
Berardo Di Matteo $^{1} \cdot$ Vittorio Tarabella $^{1} \cdot$ Giuseppe Filardo $^{1} \cdot$ Patrizia Tomba $^{2} \cdot$

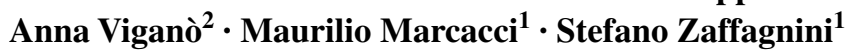

Received: 11 October 2015 / Accepted: 9 December 2015 / Published online: 30 December 2015

(C) European Society of Sports Traumatology, Knee Surgery, Arthroscopy (ESSKA) 2015

\begin{abstract}
Ankle sprains are by far the most common injuries treated by sport medicine physicians. Treatment is mainly conservative, but in some cases surgical intervention is required. The aim of the present manuscript is to give an insight into the origins and developments of ankle ligaments reconstructive surgery, underlining the fundamental steps that marked the transition from a mere conservative approach to surgical treatment options. In this historical note, the most illustrious figures who contributed to this particular field of orthopaedic practice are also acknowledged.
\end{abstract}

\section{Level of evidence $\mathrm{V}$.}

Keywords History of surgery $\cdot$ Ankle sprain $\cdot$ Ankle ligaments $\cdot$ Reconstructive techniques

\section{Introduction}

Ankle sprains are by far the most common injuries treated by sport medicine physicians. Epidemiological studies report a mean incidence of 2-7 cases of ankle sprains per 1000 individuals per year in the general population $[18,33]$. Despite being so common, the management of such injuries requires particular attention in order to

Berardo Di Matteo

berardo.dimatteo@gmail.com

1 II Orthopaedic and Traumatologic Clinic, Biomechanics and Technology Innovation Laboratory, Rizzoli Orthopaedic Institute, Via Di Barbiano, 1/10, 40136 Bologna, Italy

2 Biblioteche Scientifiche IOR, Donazione Putti, Rizzoli Orthopaedic Institute, Bologna, Italy immediately recognize complex cases and avoid serious late sequelae, such as chronic instability. Since treatment is mainly conservative, relying on the efficacy of proper rehabilitation protocols, surgical approach for ankle ligamentous injuries is less common, but there is currently a wide spectrum of techniques available to manage such lesions [25]. Several authors have proposed different methods to treat ankle instability, with an increasing attention towards ankle ligaments reconstruction throughout the whole twentieth century. From the last decades of the nineteenth century onward, several important technical revolutions have been documented in orthopaedic history, such as the first corrective osteotomy [8], the first surgical meniscal treatment $[7,9]$ and the first anterior cruciate reconstruction [10]. This period also presented a flourishing pioneering activity of surgeons in the field of ankle ligaments reconstructive surgery.

The purpose of the present manuscript is to give an insight into the origins and developments of ankle ligaments reconstructive surgery, underlining the fundamental steps that led to the shift from a pure conservative management to surgical treatment options, and also acknowledging the most illustrious figures who contributed to this particular field of orthopaedic practice.

\section{The origins of foot and ankle surgery}

The history of ankle surgery dates back to the last two decades of the nineteenth century. Before that, similar to injuries affecting knee ligaments, the therapeutic approach was just conservative and consisted of immobilization and bracing. There was also a critical difference in the type of patients. The first pioneers of foot and ankle surgery were dealing with completely different problems 


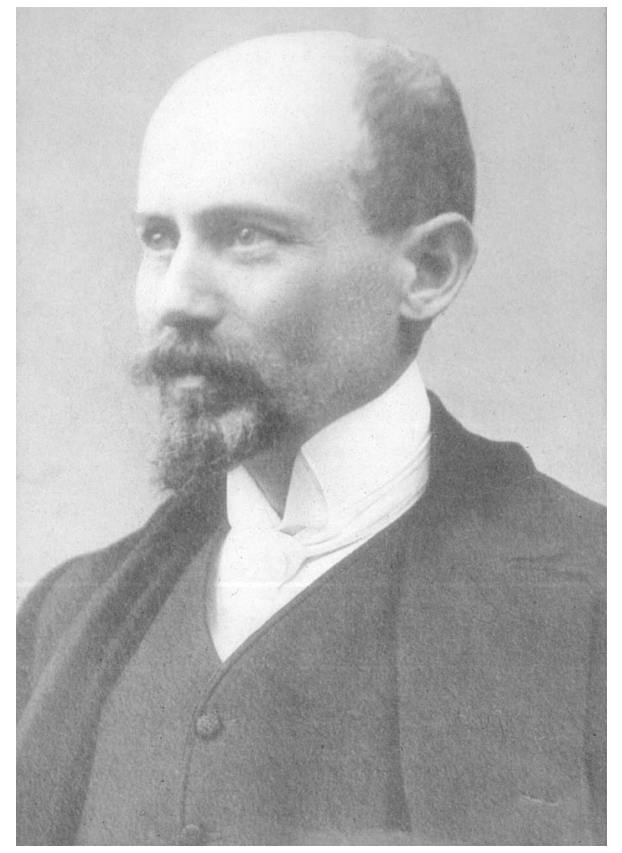

Fig. 1 Portrait of the Italian surgeon Alessandro Codivilla (18611912)

than ankle sprains in sport active patients. In fact, their main clinical interest was the correction of child deformities, both congenital and related to neurological disorders or infectious diseases. Thus, innovative techniques were tested and developed for the first time inside this particular field of paediatric orthopaedics. Tenotomies, tendon transfers and tenodesis were the strategies developed by "ancient" surgeons to manage foot deformities, both for the simplest (such as isolated pes planus) and for the most complex cases (such as paralytic clubfoot). These techniques represented the underpinnings of ankle ligaments surgery as it is intended today. The first surgeon to be remembered is Nicoladoni who, in 1880, introduced the "tendon transplantation" approach to treat a difficult case of paralytic talipes equinovarus foot [32]. Unfortunately, the clinical outcome was not satisfactory, but led to an increasing interest towards this particular approach. In later years, Parrish in 1892, Winckelmann in 1894 and Milliken in 1895, worked independently on the tendon transfer technique, with encouraging clinical outcomes. It was in 1896 that the Polish surgeon Drobnik published the first case series about 16 patients treated by this innovative surgical approach [32]. Another fundamental contribution came from the experience of Codivilla (Fig. 1), head of the Rizzoli Orthopaedic Institute in Bologna, Italy [7]. He was among the first to perform a correction of paralytic feet through the use of functional tenodesis: the tendons across the joint were isolated and detached from their distal insertion; they were then reinserted more proximally by creating a periosteal flap and a groove underneath it, where the tendon was placed before suturing the periosteum onto it. This way, the transferred tendons acted as a tie rod that could correct the deformity, thanks to the new insertion in a functional position, and could also stabilize the joint restricting (but not completely abolishing) its movements. In his paper, published in 1901 [5], Codivilla described the promising results obtained in 92 patients who underwent this particular approach, which was often associated with procedures on bones, such as osteotomies or arthrodesis, in order to provide the best functional outcome for these difficult deformities. The technique developed by Codivilla was immediately internationally recognized and was applied by several surgeons across Europe, sometimes with modifications, like the one proposed in 1912 by Vulpius, consisting in fixing the tendon beneath both periosteal and bone flaps instead of the periosteum alone [9]. A year later, the Canadian surgeon Gallie reported his personal experience with a similar "tendon fixation" approach to treat equinovarus foot deformity [13]: he employed both peroneus brevis and peroneus longus tendons in order to achieve a stable and lasting correction, with lower risk of deformity relapse over time. The overall brilliant results obtained by these innovative techniques for the treatment of foot and ankle deformities encouraged surgeons to test them in different clinical conditions. It took just a few years to see these surgical principles applied also in the field of joint instability, thus marking the beginning of the era of ankle ligaments surgery.

\section{Ankle ligaments surgery: a chronicle of fundamental achievements}

Despite sport practice being far less common at the beginning of twentieth century, traumatic injuries occurring during working activities were rather frequent, and acute and chronic instability could represent a significant cause of disability. Therefore, the correct management of this kind of injuries was perceived as paramount both by patients and by physicians. This increasing awareness led to the development of several techniques over a relatively little time span.

The first medical report concerning ankle ligaments reconstruction was published by Nilsonne in 1932 [27]. He treated a 13-year-old girl affected by a rupture of the calcaneo-fibular ligament. The surgical procedure consisted in suturing the torn ligament and reinforcing it by using the peroneus brevis tendon, which was split: the proximal portion was sutured to the peroneus longus tendon, whereas the distal one was fixed onto the previously sutured calcaneo-fibular ligament (Fig. 2). A good clinical outcome was reported at 4 months, and the talar tilt was no more 
Fig. 2 A pictorial chronicle resuming the fundamental techniques proposed for ankle ligaments reconstruction
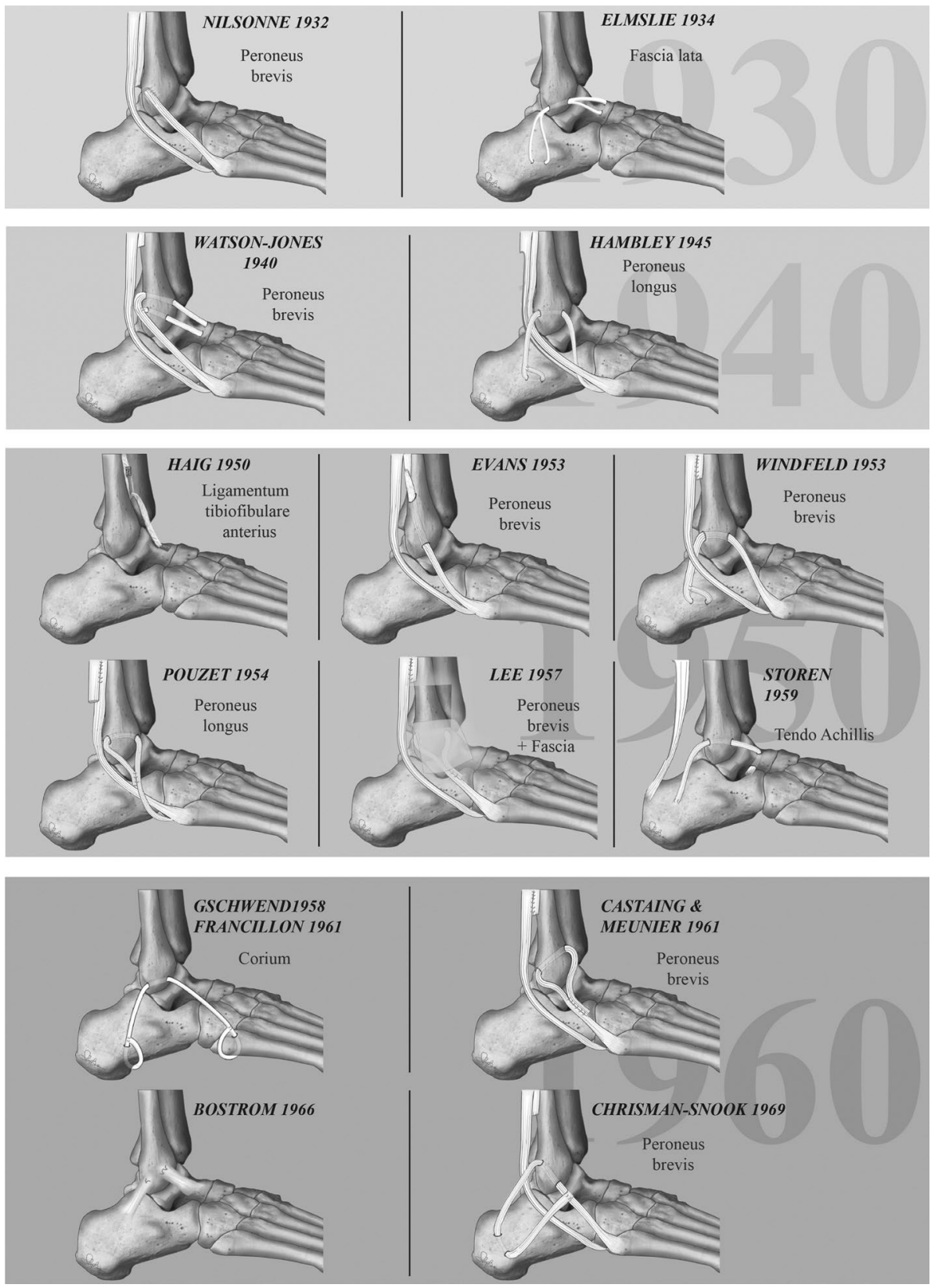

present at stress X-rays examination. A couple of years later, Emslie described his novel technique to reconstruct both the anterior talo-fibular ligament and the calcaneo-fibular ligament [11]. He employed a strip of fascia lata (harvested from the ipsilateral thigh) and then drilled a series of seven holes in the talus, lateral malleolus and calcaneus. He then passed the fascia lata graft through the holes in a precise order to reconstruct the functional complex of lateral ankle ligament (Fig. 2). Four patients were treated by this technique, all with positive results occurring within few months after the operation.

In 1940, Sir Watson-Jones (Fig. 3) proposed his own reconstructive approach, whose success is testified by the large number of surgeons who later adopted, and sometimes modified, his original technique, with long-term results well documented in the literature $[34,35]$. The technique is described in his fundamental text entitled "Fractures and other Bone and Joint Injuries" and consisted in splitting the peroneus brevis tendon retaining its distal insertion. A drill was then performed in the lateral malleolus from posterior to anterior, and the graft passed through the malleolus and then through a tunnel hollowed in the lateral aspect of the talus. The graft was then pulled backward, passed through a second tunnel in the lateral malleolus and, finally, sutured to itself in the postero-lateral aspect of the fibula (Fig. 2). A few years after the description of 


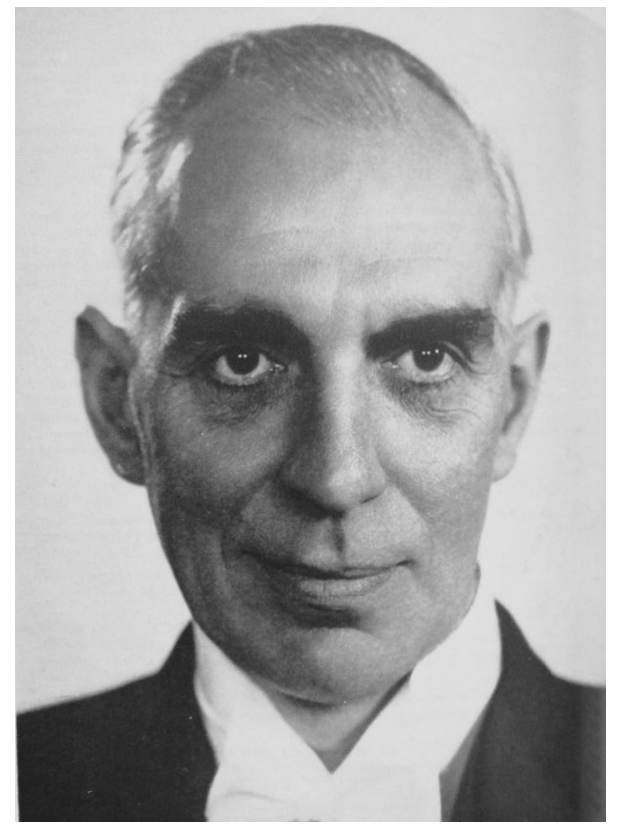

Fig. 3 Portrait of the English surgeon Sir Reginald Watson-Jones (1902-1972)

the Watson-Jones technique, the English surgeon Hambly reported a case of chronic ankle instability in a 32-year-old female, and he treated using the peroneus longus tendon that "was split from above downwards. The detached end is then threaded through the external malleolus and through the os calcis". This simple method resulted in a very satisfactory outcome [14].

The period ranging from 1940 to 1970 appears to be some sort of a "golden age" in the field of ankle ligaments reconstruction. It saw several novel reconstructive techniques flourishing, and more significantly, the debate about functional anatomy of the ankle reached significant importance in the orthopaedic discussions of that period. Beyond the techniques already mentioned, other authors contributed with their effort in this field. In 1950, Canadian surgeon Gibson proposed to shorten the peroneus brevis and peroneus tertius tendons as an effective and more physiologic approach to correct ankle instability compared to the "standard" procedures of tenodesis previously invented [17]. In the same year, another Canadian physician, Haig, was the first to use the anterior tibio-fibular ligament. He detached the tibial attachment of this ligament with a bone plug and then swung it down and fixed it with a screw into the lateral surface of the talus while the foot was held in inversion. The author reported two successful cases, but there are no traces of other surgeons adopting his original technique [16] (Fig. 2). In 1952, the well-known English surgeon Evans proposed his own technique (Fig. 2) which he described with these words: "the tendon of the peroneus brevis is divided at the upper limit of the incision. The distal end is stripped of its lowermost muscle fibers, and dissected free to the back of the lateral malleolus. Here it is withdrawn from the superior peroneal retinaculum to below and in front of the lateral malleolus. A drill hole is made diagonally forward and downward through the lower end of the fibula. The tendon is threaded back through this drill hole, and with the foot held in slight eversion, is reattached to the proximal end with any necessary overlap". Evans treated 14 patients who were followed up for up to 3.5 years, revealing overall satisfactory results [12]. In 1953, Windfeld introduced a modification to the WatsonJones technique (Fig. 2). He harvested the distal end of the peroneus brevis tendon and passed it through the fibular tunnel from anterior to posterior and not, as WatsonJones, from posterior to anterior. In this way, he believed to obtain a better tightening of the neo-ligament, which was fixed on the periosteum of the malleolus both at the entrance and exit of the fibular tunnel and to the neck of talus, without any need to drill other holes in the talus [36]. In the same year, the French surgeon Pouzet proposed his own reconstructive procedure (Fig. 2): he split the peroneus longus and made it pass through a fibular tunnel from anterior to posterior; he then pulled the graft downward to create a loop around the lateral malleolus and fixed it both to the calcaneus and to itself [29]. A further variation in the Watson-Jones technique was introduced by Lee in 1957 (Fig. 2): his method consisted in passing the peroneus brevis graft from backward to forward in a fibular tunnel (exactly as proposed by Watson-Jones) and then downward and below the lateral malleolus, where the graft was sutured to itself and to the sheath of peroneus longus. To provide a superior stabilization in inversion, a flap of fascia was harvested from the lower portion of fibula and pulled downward to be sutured onto the neo-ligament to further strengthen the construct. The author treated seven patients who were followed for up to 12 years after surgery, showing that the modified Watson-Jones was able to guarantee an excellent outcome even in the long term [24].

A corium graft was used in 1958 by the German surgeons Gschwend and Francillon to reconstruct the torn ankle ligaments. The technique was particularly skilful (Fig. 2): three tunnels were made, one in the posterior calcaneus, one in the lateral malleolus and one in the fifth metatarsal bone; the corium graft was then passed through these tunnels, and the distal ends were fixed onto the graft itself to hold the construct in site [15].

In 1959, another completely new approach was developed: for the first time, Storen used Achilles tendon to reconstruct the lateral ligaments (Fig. 2). The procedure was quite complex and technically demanding: after harvesting the medial third of the Achilles tendon, this was pulled anteriorly to the remaining portion of the tendon and 
guided subcutaneously towards the lateral malleolus. Here, two drill holes were performed: the first in the malleolus and the second in the talar neck. The Achilles graft was then passed from posterior to anterior in the fibular tunnel and then upward to downward in the talar neck tunnel. Finally, it was firmly sutured to the soft tissue in the sinus tarsi. According to the author, this procedure was able to provide a stable reconstruction of all three lateral ligaments, and in case of persisting instability, it was possible to perform a second operation (at least 4 months after the first), where the Achilles graft could be detached from its calcanear tuberosity insertion and sutured intraosseously at the site of insertion of the calcaneo-fibular ligament to further reinforce it [30]. In the same year, 1961, the "triangular ligamentoplasty" was proposed by the French surgeon Castaing (Fig. 2): he split the peroneus brevis tendon and passed it through a fibular tunnel from posterior to anterior and downward to upward; the remaining graft was then looped around the malleolus and sutured to its distal ends in a "bottle opener" fashion, with a triangular head. Eight cases were included in the study, and seven of them were classified as excellent or good at short-term evaluation [3].

In this complex scenario of different techniques, all characterized by a "non-anatomic" reconstruction of the ankle lateral ligaments, true innovative thinking came from the experience of the Swedish surgeon Brostrom, who argued that the available reconstructive techniques (in particular the Watson-Jones approach, which was the most used at the time) did not allow a restoration of normal biomechanics and led to an altered function of the talo-crural and subtalar joints [2]. Based on these facts, he was the first to propose the suture of the torn ankle ligaments in a chronic setting. In his milestone paper published in 1966, he states that “... contrary to previously expressed opinions, ruptured ankle ligaments can be exposed and sutured many years after the causal injury, and that in most cases the operation gives complete restoration of function". Brostrom was used to directly suture end-to-end torn ligaments when enough tissue was available; otherwise, when dealing with anterior talo-fibular ligament lesions very proximal to their fibular attachment, he anchored it to the anterior margin of the fibula, properly cleaned, by sutures that were drawn through holes drilled in the lateral malleolus, with further reinforcement by a flap of soft tissue to cover the reinserted ligament (Fig. 2). This novel approach encountered a large success among practitioners, and with some modifications, it is still applied in clinical practice, thus testifying the revolutionary contribution by Brostrom [19].

A few years later, Chrisman and Snook proposed a variation in the technique firstly developed by Emslie (Fig. 2). Their approach consisted in splitting longitudinally the peroneus brevis tendon. One half of the tendon was mobilized from its retinaculum and then passed through a hole drilled parallel to the articular surface in the distal fibula, from anterior to posterior. The remaining portion of the tendon is pulled down to the calcaneus where a horizontal groove is created: the tendon is placed into the groove and sutured there under moderate tension. Finally, if long enough, the hemi-tendon was sutured to its original distal end, near the fifth metatarsus. The authors treated seven athletic patients by this technique, and all of them were able to resume their sport practice at the same pre-injury level [4].

From the late 1960s onwards, ankle ligaments surgery remained on the edge of interest of orthopaedic practitioners, also due to the increasing rate in sport participation, which led to a raising incidence of both acute and chronic ligament injuries requiring surgical treatment. This particular field of sport medicine was marked by many further progresses over the last 40 years, which are based on the fundamental landmarks and pre-eminent pioneers described in this historical note.

\section{Discussion}

The main findings of the present historical note are the following: foot and ankle functional surgery started at the very end of the nineteenth century, thanks to the contribution of surgeons mainly focused on the treatment of congenital or acquired deformities; afterwards, ankle ligaments surgery has gained a constantly increasing interest over the twentieth century with a truly remarkable number of techniques proposed in this period that makes this a peculiar and unique chapter in the history of orthopaedics.

As seen also in other surgical fields, such as knee surgery [10], the first treatments were performed on very complex patients, often in association with bone procedures, to correct deformities. Therefore, the typical patient was not the athlete who got injured during sport practice, but rather a person affected by a severe disease compromising the entire foot. The necessity of addressing the complex needs of this kind of patients stimulated surgeons to find innovative approaches that could guarantee the best clinical outcome. In this perspective, the challenging conditions treated represented the premise for developing techniques that would have been later applied in the management of less difficult but more common cases.

The second fundamental aspect to consider is the understanding of ankle biomechanics and the deleterious consequences of a failed conservative treatment in terms of patients' quality of life and working ability. It was no more acceptable that patients were doomed to chronic pain and functional limitation after conservative management failure, and this stimulated the development of new surgical approaches to restore function and provide symptomatic relief in patients affected by chronic ankle 
instability. This awareness can be regarded as another remarkable feature of medical progress, i.e. the close correlation between technical advancements and the definition of the correct indications for treatments. In fact, it is not just the evolution in surgical techniques that leads to a better outcome, but also the understanding of when and how the proper treatment is performed, according to the specific injury pattern and patients' features. When the pioneers of foot and ankle surgery opened the path of ankle ligaments reconstruction, there was no established treatment algorithm for managing these lesions. The initial treatment was always conservative, and operative management was considered, based on clinical examination and stress X-rays, only by a selected number of physicians for those patients complaining chronic instability and severe functional limitation: they were true forerunners and their approach can be considered still "modern" according to the most recent standards. The main problem was that there were just a few surgeons capable of giving the right indication to surgery and performing it. Both the progress in the knowledge of anatomy, physiology and biomechanics of the foot and ankle district, and the sharing of the first clinical experiences by the pioneers in this field contributed in establishing ankle ligaments surgery as a viable treatment option for ankle instability. At the same time, further studies helped to understand and optimize also the conservative management for such lesions, thus leading to the definition of guidelines [21] to help sport medicine physicians in the choice of the best option for their patients. Currently, the most recent indications suggest that, in the general population, acute ankle ligaments injuries should be initially managed by proper conservative means [20], and in case of failure with persisting chronic ankle instability, surgery should be considered. However, in particular cases, especially in the athletic high-demanding patients, surgery can be regarded as a first-line treatment to restore proper ankle stability and guarantee early return to sport practice [20, 22].

Coming back to the history of ankle ligaments surgery, it is also extremely fascinating to see the great number of contributions to this field of surgery coming from all over the world in a time span of $<40$ years (from 1930 to the late 1960s). The ingenuity of the invented techniques is also truly surprising. The majority of surgeons adopted tenodesis using peroneal tendons, but also Achilles tendon and corium were tested. The approach made by Brostrom was revolutionary [19]: he was the first to firmly believe in the possibility of suturing or re-anchoring chronically injured ligaments, thus marking a drastic (and still actual) change in the management of such lesions. From that period onward, other amazing progresses have been reached. Some of the "historical" techniques here described have been improved and are still applied [19, 26]; the use of allografts has been made possible due to the establishment of musculo-skeletal tissue banks [6]; novel fixation devices have been introduced to improve the stability of the reconstruction [28]; arthroscopy helped to reduce the invasiveness of the procedures [1]; finally, new biological augmentation strategies are currently tested to enhance tissue healing $[23,31]$.

The current wide spread of ankle surgery and the presence of some of the described technical contributions in current orthopaedic practice further allow to stress the importance of the efforts made by the pioneers mentioned in this historical note.

\section{Conclusions}

The evolution occurred in the field of ankle ligaments surgery is one of the most fascinating chapters in the history of orthopaedics. Across the twentieth century, many surgeons have proposed original contributions, some of them encountering long-lasting success. Medical progress in this particular area of sport traumatology is still running fast, and what has been reported in the present manuscript can be regarded as the pillars on which modern foot and ankle surgery is standing.

Acknowledgments The authors would like to thank Liliana Draghetti (Donazione Putti, Biblioteche Scientifiche, Rizzoli Orthopaedic Institute), Silvia Bassini (Biomechanics Lab, Rizzoli Orthopaedic Institute) Davide Puppini, Lucia Mancini and Linda Accorsi for their help. The present paper was supported by the following funds granted to Rizzoli Orthopaedic Institute: “5 \%o, anno 2012”.

\section{Compliance with ethical standards}

Conflict of interest The authors declare that they have no conflict of interest with respect to the contents of the present manuscript.

\section{References}

1. Acevedo J, Mangone P (2015) Ankle instability and arthroscopic lateral ligament repair. Foot Ankle Clin 20:59-69

2. Broström L (1966) Sprained ankles. VI. Surgical treatment of "chronic" ligament ruptures. Acta Chir Scand 132:551-565

3. Castaing J, Le Chevallier P, Meunier M (1961) Entorse a repetition ou subluxation recidivante de la tibio-tarsienne. Une technique simple de ligamentoplastie externe. Rev Chir Orthop 27:598-608

4. Chrisman O, Snook G (1969) Reconstruction of lateral ligament tears of the ankle. An experimental study and clinical evaluation of seven patients treated by a new modification of the Elmslie procedure. J Bone Joint Surg Am 51:904-912

5. Codivilla A (1901) Sul trattamento delle deformità paralitiche nei bambini. In: Putti V (ed) Scritti Medici. Licinio Cappelli, Bologna, pp 462-472

6. Dierckman B, Ferkel R (2015) Anatomic reconstruction with a semitendinosus allograft for chronic lateral ankle instability. Am J Sports Med 43:1941-1950 
7. Di Matteo B, Moran C, Tarabella V, Viganò A, Tomba P, Marcacci M, Verdonk R (2015) A history of meniscal surgery: from ancient times to the twenty-first century. Knee Surg Sports Traumatol Arthrosc. doi:10.1007/s00167-015-3717-2

8. Di Matteo B, Tarabella V, Filardo G, Viganò A, Tomba P, Marcacci M (2013) John Rhea Barton: the birth of osteotomy. Knee Surg Sports Traumatol Arthrosc 21:1957-1962

9. Di Matteo B, Tarabella V, Filardo G, Viganò A, Tomba P, Marcacci M (2013) Thomas Annandale: the first meniscus repair. Knee Surg Sports Traumatol Arthrosc 21:1963-1966

10. Di Matteo B, Tarabella V, Filardo G, Tomba P, Viganò A, Marcacci M, Zaffagnini S (2015) Knee multi-ligament reconstruction: a historical note on the fundamental landmarks. Knee Surg Sports Traumatol Arthrosc. doi:10.1007/s00167-015-3615-7

11. Elmslie R (1934) Recurrent subluxation of the ankle joint. Ann Surg 100:364-367

12. Evans D (1953) Recurrent instability of the ankle. A method of surgical treatment. Proc R Soc Med 46:343-344

13. Gallie W (1913) Tendon fixation-an operation for the prevention of deformity in infantile paralysis. Am J Orthop Surg 211:151-155

14. Gibson A (1950) Operation for recurrent subluxation of the ankle joint. J Bone Joint Surg 32B:751

15. Gschwend N (1958) Die fibularen bandlesionen mit der haufig verkannten folge der fussverstauchungen. Praxis 47:809-813

16. Haig H (1950) Repair of ligaments in recurrent subluxation of the ankle joint. J Bone Joint Surg 32B:751-755

17. Hambley E (1945) Recurrent dislocation of ankle due to rupture of external lateral ligament. Br Med J 1:413

18. Hølmer P, Søndergaard L, Konradsen L, Nielsen P, Jørgensen L (1994) Epidemiology of sprains in the lateral ankle and foot. Foot Ankle Int 15:72-74

19. Karlsson J, Eriksson BI, Bergsten T, Rudholm O, Swärd L (1997) Comparison of two anatomic reconstructions for chronic lateral instability of the ankle joint. Am J Sports Med 25:48-53

20. Kerkhoffs GM, Handoll HH, de Bie R, Rowe BH, Struijs PA (2007) Surgical versus conservative treatment for acute injuries of the lateral ligament complex of the ankle in adults. Cochrane Database Syst Rev 18(2):CD000380

21. Kerkhoffs GM, van den Bekerom M, Elders LA, van Beek PA, Hullegie WA, Bloemers GM, de Heus EM, Loogman MC, Rosenbrand KC, Kuipers T, Hoogstraten JW, Dekker R, Ten Duis HJ, van Dijk CN, van Tulder MW, van der Wees PJ, de Bie RA (2012) Diagnosis, treatment and prevention of ankle sprains: an evidence-based clinical guideline. Br J Sports Med 46(12):854-860
22. Kerkhoffs GM, Van Dijk CN (2013) Acute lateral ankle ligament ruptures in the athlete: the role of surgery. Foot Ankle Clin $18(2): 215-218$

23. Laver L, Carmont MR, McConkey MO, Palmanovich E, Yaacobi E, Mann G, Nyska M, Kots E, Mei-Dan O (2014) Plasma rich in growth factors (PRGF) as a treatment for high ankle sprain in elite athletes: a randomized control trial. Knee Surg Sports Traumatol Arthrosc. PMID: 24938396

24. Lee HG (1957) Surgical repair in recurrent dislocation of the ankle joint. J Bone Surg 39A:828-834

25. McCriskin BJ, Cameron KL, Orr JD, Waterman BR (2015) Management and prevention of acute and chronic lateral ankle instability in athletic patient populations. World J Orthop 6:161-171

26. Morelli F, Perugia D, Vadalà A, Serlorenzi P, Ferretti A (2011) Modified Watson-Jones technique for chronic lateral ankle instability in athletes: clinical and radiological mid- to long-term follow-up. Foot Ankle Surg 17:247-251

27. Nilsonne H (1932) Making a new ligament in ankle sprain. J Bone Joint Surg 14A:380-381

28. Paden MH, Stone PA, McGarry JJ (1994) Modified Broström lateral ankle stabilization utilizing an implantable anchoring system. J Foot Ankle Surg 33:617-622

29. Pouzet E (1954) Plastie ligamentaire externe de la tibiotarsienne. Lyon Chir 49:618-619

30. Storen H (1959) A new method for operative treatment of insufficiency of the lateral ligaments of the ankle joint. Acta Chir Scand 117:501-509

31. Vannini F, Di Matteo B, Filardo G, Kon E, Marcacci M, Giannini S (2014) Platelet-rich plasma for foot and ankle pathologies: a systematic review. Foot Ankle Surg. 20(1):2-9

32. Vulpius O (1912) Treatment of infantile paralysis. Wood, Oxford, pp 70-71

33. Waterman BR, Owens BD, Davey S, Zacchilli MA, Belmont PJ (2010) The epidemiology of ankle sprains in the United States. J Bone Joint Surg Am 92:2279-2284

34. Watson-Jones R (1940) Fractures and other bone and joints injury. E \& S Livingstone Ltd, Edinburgh

35. Watson-Jones R (1952) Recurrent forward dislocation of the ankle joint. J Bone Joint Surg 34B:519

36. Windfield P (1953) Treatment of undue mobility of the ankle joint following severe sprain of the ankle with avulsion of the anterior and middle bands of the lateral ligament. Acta Chir Scand 105:299-304 